\title{
An advance notification letter increases participation in colorectal cancer screening
}

\author{
S R Cole, A Smith, C Wilson, D Turnbull, A Esterman and G P Young
}

J Med Screen 2007; 14:73-75

See end of article for authors' affiliations

.............

Correspondence to: S R

Cole, Bowel Health Service,

Repatriation General

Hospital, Daws Road,

Daw Park,

South Australia 5041

scoles@rgh.sa.gov.au

Accepted for publication 23 March 2007

\begin{abstract}
Objectives To determine the impact of novel invitation strategies on population participation in faecal immunochemical test (FIT)-based colorectal cancer (CRC) screening.

Setting A community screening programme in Adelaide, South Australia.

Methods In total, 2400 people aged 50-74 years were randomly allocated to one of four CRC screening invitation strategies: (a) Control: standard invitation-to-screen letter explaining risk of CRC and the concept, value and method of screening; (b) Risk: invitation with additional messages related to $C R C$ risk; (c) Advocacy: invitation with additional messages related to advocacy for screening from previous screening programme participants and (d) Advance Notification: first, a letter introducing Control letter messages followed by the standard invitation-to-screen. Invitations included an FIT kit. Programme participation rates were determined for each strategy relative to control. Associations between participation and sociodemographic variables were explored.

Results At 12 weeks after invitation, participation was: Control: $237 / 600$ (39.5\%); Risk: 242/600 (40.3\%); Advocacy: $216 / 600$ (36.0\%) and Advance Notification: 290/600 (48.3\%). Participation was significantly greater than Control only in the Advance Notification group (Relative risk [RR] 1.23, 95\% confidence interval [Cl] 1.06-1.43). This effect was apparent as early as two weeks from date of offer; Advance Notification: $151 / 600$ (25.2\%) versus Control: 109/600 (18.2\%, RR 1.38, 95\% Cl 1.11-1.73).

Conclusions Advance notification significantly increased screening participation. The effect may be due to a population shift in readiness to undertake screening, and is consistent with the Transtheoretical Model of behaviour change. Risk or lay advocacy strategies did not improve screening participation. Organized screening programmes should consider using advance notification letters to improve programme participation.
\end{abstract}

\section{INTRODUCTION}

$\mathrm{P}$ opulation screening by guaiac faecal occult blood testing (FOBT) reduces mortality from colorectal cancer $(\mathrm{CRC})^{1-3}$ and reduces incidence of $\mathrm{CRC}$ through removal of colorectal adenomas. ${ }^{4}$ FOBTs are ideally suited for population screening for CRC because screening can be implemented through the postal system. Therefore, effective print-based communication between screening providers and programme invitees is crucial for high levels of uptake, although few studies have investigated the direct impact of alternative invitation strategies on screening programme participation.

Actual participation rates in FOBT-based population screening ${ }^{5}$ have been well below the estimated $75 \%$ for whom FOBT-based screening is medically appropriate. ${ }^{6}$ In studies exploring ways to improve participation in FOBTbased screening, we identified three strategies that had the potential to improve programme uptake over a standard invitation: (1) Enhanced awareness of CRC risk; (2) Advocacy for screening and (3) Advance notification of invitation.

\section{Enhanced awareness of risk for CRC}

Perceptions of risk and vulnerability are powerful determinants of participation in screening, although the effect may be modulated by how risk is expressed. ${ }^{7}$ Perceived risk for
CRC has previously been shown to influence uptake in FOBT screening, ${ }^{8}$ and an improved understanding of personal risk is associated with uptake of mammography screening. ${ }^{9}$

\section{Advocacy for screening}

Celebrity advocacy has been shown to promote uptake of health programmes, including cancer screening, ${ }^{10}$ but the strategy suffers from being opportunistic and often has only temporary effects. ${ }^{11}$ General practitioner advocacy for screening improved uptake among patients, ${ }^{12}$ but can be difficult to apply on a population scale. However, statements supporting screening from individuals of similar age and outlook to programme invitees, especially from those with previous successful screening outcomes, could enhance uptake of screening.

\section{Advance notification of invitation}

Previous FOBT screening trials have used the strategy of sending an advance notification letter alerting recipients that an invitation to screen for CRC will soon follow. ${ }^{13-17}$ While most studies showed that this was associated with increased uptake, it was often administered together with educational materials and so the effect alone is unclear. Advance notification has never been trialled in programmes 
using faecal immunochemical tests (FIT) where several barriers to participation are reduced. ${ }^{11}$

The aim of this study was to determine the impact of three novel letter-based invitation-to-screen strategies on participation in FIT-based CRC screening.

\section{METHODS}

\section{Invitation letter content}

Control

A standard invitation letter containing health-related messages concerning CRC prevention and the value and ease of screening.

\section{Risk}

An invitation containing the standard invitation messages, plus additional positively framed messages about CRC risk, generalized risk for CRC and relative risk for CRC.

\section{Advocacy}

An invitation containing the standard invitation messages, plus messages from 'like' others advocating CRC screening. Colour photographs of the lay advocates (one male, one female) and personal endorsement statements were included.

\section{Advanced notification}

First, an Advance Notification letter introducing standard invitation messages, followed in two weeks by the standard (Control group) invitation.

\section{Study population}

Invitees were selected from the electoral roll of the Australian Electoral Commission (AEC). The extract consisted of the names and addresses of 24,905 people aged 50-74 years on 29 October 2004 from nine postcode areas. In all, 2400 people were randomly selected and allocated to Control, Risk, Advocacy and Advance Notification groups.

\section{Screening implementation}

Between 17 January and 7 April 2005, those selected were mailed an offer of FIT screening from a central screening facility located within a public hospital. The offer included an invitation letter, study information, an InSure ${ }^{\circledR}$ FIT kit (Enterix Australia Inc.) and a reply paid envelope. All invitation letters were single page printed on hospital letterhead. Invitees were sent a reminder letter at six weeks from invitation if required. FIT sample cards were developed by an accredited pathology laboratory. All test positive participants were encouraged to complete colonoscopy follow-up.

\section{Outcome measures}

The primary outcome was participation in screening, defined as the return of a completed sample card within 12 weeks from the date screening offers were posted. Secondary outcomes were participation at two weeks from the date invitations were mailed (early participation), and participation at 14 weeks from first contact, defined as
14 weeks from date of mailing the first print-based communication.

\section{Other data}

The AEC supplied age (as 5-year band), sex and suburb in addition to name and street address data. Knowledge of suburb allows the index of relative socioeconomic disadvantage (IoSED, Australian Bureau of Statistics) to be determined. Disadvantaged areas have low IoSED values.

\section{Statistical analyses}

FIT sample card returns were monitored for 14 weeks from the date the screening invitation was sent. Intervention groups were similar for proportions of sex, age band and IoSED. Unadjusted comparisons of participation by group and sociodemographic variables were undertaken using univariate generalized linear models (GLM). Comparisons of participation by group adjusted for sociodemographic variables were undertaken using multivariate GLM. Clustering by suburb was adjusted for in all models. All analyses were undertaken using the statistical software STATA 8 (StataCorp).

\section{RESULTS}

\section{Impact of invitation strategy on participation}

The Advance Notification group had a significantly increased participation rate compared with the Control group at week 12 (univariate GLM relative risk $(\mathrm{RR})=1.23,95 \%$ confidence interval [CI] 1.06-1.43). The same pattern was seen at week 2 with an even stronger relationship (Advance Notification: 151/600 [25.2\%] versus Control: 109/600 (18.2\%), RR 1.38, 95\% CI 1.11-1.73). Adjusting for sex, age group and IoSED provided similar results for week 12, but reduced the impact of Advanced Notification at week 2. The Risk and Advocacy strategies failed to increase participation relative to the Control (Week 12 results: Risk; 242 sample cards returned/600, 40.3\% participation rate, Advocacy; 216/600, 36.0\%).

\section{Adjustment for 2-week difference in programme awareness}

The Advance Notification group had had an extra two weeks' awareness about the screening programme at week 12 compared with the Control group, which may have contributed to their better overall participation. We therefore compared participation at 14 weeks from first contact, where participation was: Control 238/600, 39.7\%; Advance Notification 290/600, 48.3\%, unadjusted and adjusted $\mathrm{RR}=1.22,95 \%$ CI $1.05-1.42)$.

\section{Associations with participation}

Age was associated with participation at both week 12 (age band 60-64 years: RR 1.32, CI 1.12-1.56; 65-69 years: RR 1.47, CI 1.23-1.77) and week 2 (60-64 years: RR 1.50, CI 1.13-2.01, 65-69 years: RR 1.76, CI 1.36-2.27). Being female was associated with participation at week 12 (RR 1.13, CI 1.03-1.26). IoSED was weakly associated with participation at week $12(P=0.067)$ with more advantaged suburbs having higher participation. 


\section{DISCUSSION}

These results demonstrate that a simple advance notification letter had a significant and positive effect on participation in FIT-based CRC screening. The effect was large and immediate. In contrast, two other interventions were ineffective in promoting participation levels above that of a standard invitation letter.

Advance notification letters were used in early trials of guaiac FOBT screening. ${ }^{14-17}$ However, those studies failed to show a consistent increase in participation, perhaps because advance notification letters were accompanied by educational material or the effect was masked by other barriers to participation associated with guaiac FOBTs. Using an advance notification letter with low information burden, coupled with removal of diet and medication barriers through the use of immunochemical tests, may allow the positive effect of advance notification to become evident.

The difference in participation between the Advance Notification and Control groups was not due to lead-time bias because, when participation was monitored for 14 weeks from initial contact, the difference in participation between Control and Advance Notification groups remained constant and significant.

Early gains in knowledge of the magnitude, risk and curability of CRC due to the advance notification letter, reinforced by similar information in the standard invitation letter, may be responsible for the immediate effect of this strategy. This is of importance to screening programme planning, where the test return pattern determines reminder letter and test development workloads. A rapid response to a screening invitation, following an advance notification letter, at least partly, balances an overall increase in programme postage costs due to reduced reminder costs.

It is unclear why invitations that included risk and advocacy messages failed to improve participation. To ensure that additional messages did not adversely influence the comprehension level, we kept the simple measure of gobbledegook scores ${ }^{18}$ to within one grade level. However, it is possible that slightly increased length of the risk and advocacy invitations may have contributed to information burden, negating any effect on participation. The Advance Notification group received an invitation letter identical to the Control group and would not have been affected by information burden.

In univariate analyses, we demonstrated associations between participation at 12 weeks and 2 weeks and age, and between participation at 12 weeks and being female. The strongest association was between participation at two weeks and being aged 65-69 years. Low uptake in the youngest age band is likely to be due to perceived lack of time and low perceived risk. Developing interventions to specifically improve participation in the younger age bands should be a priority.

Health behaviour change models, such as the Transtheoretical Model (TTM) and its variants, propose that health behaviours follow a 'stages of change' pathway. ${ }^{19}$ Our findings are consistent with the TTM and support the notion that an advance notification letter moves people from being unaware of CRC or CRC screening to being aware, or from being aware to contemplation of action, so that when an actual offer of screening that includes a screening test is made, it is only a small step further to complete the required behaviour.

These results highlight the potential benefit of a simple advance notification letter in population-based CRC screening programmes.
This study was funded by a grant from the National Health and Medical Research Council of Australia. Screening tests were donated by Enterix Aust Pty Ltd.

Trial registration. Australian Clinical Trials Registry: ACTRN012605000239662.

\section{Authors' affiliations}

S R Cole, Principal Medical Scientist, Bowel Health Service, Repatriation General Hospital Daw Park, Daw Park, South Australia 5041, Australia

A Smith, Research Associate, Department of Medicine, Flinders University of South Australia, Bedford Park, South Australia 5042, Australia

C Wilson, Principal Research Scientist, Division of Human Nutrition, CSIRO, Adelaide, South Australia 5000, Australia and CSIRO Preventative Health National Research Flagship

D Turnbull, Professor, School of Psychology, University of Adelaide, Adelaide, South Australia 5005, Australia

A Esterman, Professor, Division of Health Sciences, School of Nursing and Midwifery, University of South Australia, Adelaide, South Australia 5000, Australia

G P Young, Professor, Department of Medicine, Flinders University of South Australia, Bedford Park, South Australia 5042, Australia

\section{REFERENCES}

1 Mandel JS, Bond JH, Church TR, et al. Reducing mortality from colorectal cancer by screening for fecal occult blood. $N$ Engl J Med 1993;329: 1365-71

2 Hardcastle JD, Chamberlain JO, Robinson $\mathrm{MH}$, et al. Randomised controlled trial of faecal-occult-blood screening for colorectal cancer. Lancet 1996;348: 1472-7

3 Kronborg $\mathrm{O}$, Fenger $\mathrm{C}$, Olsen J, et al. Randomised study of screening for colorectal cancer with faecal-occult-blood test. Lancet 1996:348: 1467-1471

4 Mandel JS, Church TR, Bond JH, et al. The effect of fecal occult-blood screening on the incidence of colorectal cancer. N Engl J Med 2000;343: 1603-1607

5 Young GP, Rozen P, Levin B. How should we screen for early colorectal neoplasia?. In: Rozen P, Young GY, Levin B, et al. (eds) Colorectal Cancer in Clinical Practice: Prevention, Early Detection and Management. 2nd edn. Taylor and Francis: Abingdon, 2006;97-130

6 Subramanian S, Klosterman M, Amonkar MM, Hunt TL. Adherence with colorectal cancer screening guidelines: a review. Prev Med 2004;38: $536-550$

7 Sarfati D, Howden-Chapman P, Woodward A, Salmond C. Does the frame affect the picture? A study into how attitudes to screening for cancer are affected by the way benefits are expressed. J Med Screen 1998:5:137-40

8 Macrae FA, Hill DJ, St John DJB, et al. Predicting colon cancer screening behavior from health beliefs. Prev Med 1984;13:1 15-26

9 Edwards A, Unigwe S, Elwyn G, et al. Personalised risk communication for informed decision making about entering screening programs. Cochrane Database Syst Rev 2003;(1):D001865

10 Larson R, Woloshin S, Schwartz LM, et al. Celebrity endorsements of cancer screening. J Natl Cancer Inst 2005;97:693-5

11 Cram P, Fendrick AM, Inadomi J, et al. The impact of a celebrity promotional campaign on the use of colon cancer screening: The Katie Couric effect. Arch Int Med 2003;163:1601-5

12 Cole SR, Young GP, Byrne D, et al. Participation in screening for colorectal cancer based on a faecal occult blood test is improved by endorsement by the primary care practitioner. J Med Screen 2002;9:147-52

13 Myers RE, Ross E, Jepson C, et al. Modeling adherence to colorectal cancer screening. Prev Med 1994;23: 142-51

14 Hardcastle JD, Armitage NC, Chamberlain J, et al. Fecal occult blood screening for colorectal cancer in the general population. Results of a controlled trial. Cancer 1986;58:397-403

15 Pye G, Christie M, Chamberlain JO, et al. A comparison of methods for increasing compliance within a general practitioner based screening project for colorectal cancer and the effect on practitioner workload. J Epidemiol Commun Health 1988;42:66-71

16 Myers RE, Ross EA, Wolf $\mathrm{T}$, et al. Behavioral interventions to increase adherence in colorectal cancer screening. Med Care 1991;29: 1039-1050

17 King J, Fairbrother G, Thompson C, et al. Colorectal cancer screening: optimal compliance with postal faecal occult blood test. Aust NZ J Surg 1992:62:714-19

18 McLaughlin G. SMOG grading: a new readability formula. J Reading 1969; 12:639-46

19 Prochaska JO, Velicer WF. The transtheoretical model of health behavior change. Am J Health Promot 1997; 12:38-48 